Research Article

\title{
Genome-Wide Identification and Characterization of the ALOG Domain Genes in Rice
}

\author{
Na Li, ${ }^{1}$ Yang Wang, ${ }^{1}$ Jing $L u^{2}$ and Chuan Liu $\mathbb{D}^{1,3}$ \\ ${ }^{1}$ State Key Laboratory of Hybrid Rice, College of Life Sciences, Wuhan University, Wuhan 430072, China \\ ${ }^{2}$ College of Food Science and Technology, Huazhong Agricultural University, 430070 Wuhan, China \\ ${ }^{3}$ Chongqing Key Laboratory on Big Data for Bio Intelligence, Chongqing University of Posts and Telecommunications, \\ Chongqing 400065, China
}

Correspondence should be addressed to Chuan Liu; liuchuan@whu.edu.cn

Received 7 August 2018; Accepted 6 January 2019; Published 24 February 2019

Academic Editor: Michael Nonnemacher

Copyright (c) $2019 \mathrm{Na}$ Li et al. This is an open access article distributed under the Creative Commons Attribution License, which permits unrestricted use, distribution, and reproduction in any medium, provided the original work is properly cited.

\begin{abstract}
The ALOG domain genes, named after the Arabidopsis LSH1 and Oryza G1 (ALOG) proteins, have frequently been reported as key developmental regulators in rice and Arabidopsis. However, the investigation of the ALOG gene family is limited. Here, we conducted a genome-wide investigation of the ALOG gene family in rice and six other species. In total, eighty-four ALOG domain genes were identified from the seven species, of which fourteen ALOG domain genes (OsG1/G1Ls) were identified in the rice genome. The fourteen OsG1/G1Ls were unevenly distributed on eight chromosomes, and we found that eight segmental duplications contributed to the expansion of OsG1/G1Ls in the rice genome. The eighty-four ALOG family genes from seven species were classified into six clusters, and the ALOG domain-defined motifs 1,2, and 3 were highly conserved across species according to the phylogenetic and structural analysis. However, the newly identified motifs 4 and 5 were only present in monocots, indicating a specified function in monocots. Moreover, OsG1/G1Ls exhibited tissue-specific expression patterns. Coexpression analysis suggested that OsG1 integrates OsMADS50 and the downstream MADS-box genes, such as OsMADS1, to regulate the development of rice inflorescence; OsG1L7 potentially associates with OsMADS22 and OsMADS55 to regulate stem elongation. In addition, comparative expression analysis revealed the conserved biological functions of ALOG family genes among rice, maize, and Arabidopsis. These results have shed light on the functional study of ALOG family genes in rice and other plants.
\end{abstract}

\section{Introduction}

Rice (Oryza sativa) is one of the most important crops as a sustenance source to over 3.5 billion individuals of the global population [1]. Unraveling the molecular regulatory mechanisms of diverse agronomic traits is beneficial for rice breeding practice and may be critical for global food security. In addition, rice is an ideal model organism for plant functional genomics research and studies on the evolutionary lineage of monocotyledons. Based on these prerequisites, functional genomics technologies have rapidly been developed and applied for the identification of thousands of genes controlling important agronomic traits in rice. As the online genomic data and the number of cloned genes have increased, the genome-wide analysis of gene families has become a tool to explore the potential roles of the previously uncharacterized genes in rice as well as other commercial crops [2-6].

Rice yield and yield-related traits have been considered important agronomic traits during hybrid breeding practice, particularly grain size, grain number, flowering time, and plant height. Quantitative trait loci (QTL) or genes, such as Ghd7, OsPRR37, and DTH8, were shown to affect rice yield by pleiotropically regulating rice flowering time, plant height, and grain number [7-11]. Other QTL or genes controlling grain size, including GS3, GS5, GW2, GW5, GW7, and GL3.3, can affect rice yield mainly because grain size is typically associated with grain weight [12-17]. Furthermore, a recent study established a G-protein pathway comprising five subunits of the heterotrimeric $G$ proteins that determines the grain size in rice, demonstrating a conserved system 
among angiosperms to manipulate grain/organ size [18]. Additionally, the increase in the size of lemma/palea could also potentially increase rice yields by increasing the grain size [19]. Therefore, characterization of the genes and gene homologs that regulate the shape of lemma/palea is helpful for genetically manipulating the grain size and yield-related traits.

A single rice spikelet contains a pair of subtended lemma and palea and two sterile lemmas. Several studies have reported the genes involved in the developmental regulation of lemma/palea and sterile lemma in rice. Among these genes, OsG1 represses the homeotic transformation of the sterile lemma to the regular lemma [20], and $\mathrm{TH} 1 / \mathrm{BH} 1 /$ AFD1 affects the development of the lemma/palea, spikelet morphogenesis, grain shape, and yield in rice [21-25]. Coincidentally, these two genes are both DUF640 domaincontaining proteins. The DUF640 domain is also known as the ALOG domain, named after the Arabidopsis LSH1 and Oryza G1 proteins [20, 26, 27]. However, Arabidopsis LSH1 mediates the light-dependent regulation of the hypocotyl length, appearing to have a function different from that of OsG1 [26]. In addition, TAWAWA1, another homolog of $O s G 1$, is a unique regulator of meristem activity in rice and regulates inflorescence development through the promotion of inflorescence meristem activity and the suppression of the phase change to spikelet meristem identity [28]. These results indicated that ALOG domain genes play multiple roles in plant development, especially in the developmental regulation of floral organ identity and inflorescence meristem activity. Genome-wide expression and evolutionary analyses of the ALOG domain gene family in rice and other plant species would help to understand the regulatory mechanism of this gene family; however, these studies are still limited.

In the present study, the ALOG domain genes were first identified from 7 species, including four monocotyledons (Oryza sativa, Zea mays, Sorghum bicolor, and Brachypodium distachyon), two dicotyledons (Arabidopsis thaliana and Populus trichocarpa), and a moss (Physcomitrella patens). We primarily investigated the evolutionary relationships, gene duplication, gene structure, and expression profiles of the ALOG domain gene family, particularly the ALOG domain genes $(O s G 1 / G 1 L s)$ in rice. Based on the results, we attempted to reveal the tissue-specific expression patterns and potential functions of the ALOG family genes in rice as well as in other species. Particularly, the functional relationships between OsG1/G1Ls and MADS-box genes were deduced by coexpression analyses in rice. In addition, a comparative expression analysis was applied to study the conserved functions of ALOG family genes among rice, maize, and Arabidopsis. The present study not only provides theoretical evidence for the functional study of ALOG domain genes but also evokes a further application of the ALOG domain genes in the breeding of high-yield rice and other crops.

\section{Materials and Methods}

2.1. Identification of ALOG Domain Genes. Local databases, including protein sequences, coding sequences (CDS), and
mRNA sequences, were established by downloading data from the Rice Genome Annotation Project (RGAP, release 7.0, http://rice.plantbiology.msu.edu), the Arabidopsis Information Resource (TAIR, release 10, http://www.arabidopsis .org), the Maize Genetics and Genomics Database (MaizeGDB, B73RefGen_v2, http://www.maizegdb.org), and JGI (http://genome.jgi-psf.org). HMMER (http://hmmer.org/) and name searches were performed on the local proteome sequence database by using the Pfam profile PF04852, and the results were then combined with BLAST search results to identify rice and maize ALOG domain-containing genes. Since the ALOG domain was conserved and specific, an $E$ value of $1 e-10$ was used as the cut-off in the HMMER and BLAST searches. Within the search results, we manually removed the number of hits that only had partial ALOG domains. Information on the gene structures, full-length cDNAs, and RAP accession numbers for each gene and the characteristics of the corresponding proteins were procured from RAP-DB, RGAP, and KOME (https://dbarchive. biosciencedbc.jp/en/kome/desc.html). Additionally, the Pfam (http://www.sanger.ac.uk/Software/Pfam/) and InterPro (http:// www.ebi.ac.uk/interpro/) protein family databases were used to confirm and classify each predicted ALOG protein.

2.2. Chromosomal Location and Gene Duplication Analysis. All OsG1/G1Ls were mapped onto the chromosomes by identifying their chromosomal positions in the TIGR database, and the segmentally duplicated genes were identified from the Plant Genome Duplication Database (PGDD) with a parameter of $500 \mathrm{~kb}$ [29]. The DAGchainer program was then used to confirm the segmental duplications with the parameters $V=5, B=5, E=1 e-10$ filter seg, and distan$\mathrm{ce}=500 \mathrm{~kb}$; genes separated by five or fewer genes were considered tandem duplicates [30]. The $\mathrm{Ka}$ and $\mathrm{Ks}$ values of the duplicated gene pairs were obtained from the identification results of segmentally duplicated genes in PGDD [29]. The approximate date of occurrence of the duplication events was calculated by the equation $T=\mathrm{Ks} / 2 \lambda$. The mean synonymous substitution rate $(\lambda)$ for rice is $6.5 e-9$ [31].

2.3. Phylogenic and Structural Analysis. For the phylogenetic analysis, we only considered the amino acid sequences of the ALOG domain because no other regions could be unambiguously aligned for all of the available sequences. MUSCLE software was used to align the sequences with the default parameters, and poorly aligned positions and divergent regions were eliminated from the aligned sequences by using Gblocks $[32,33]$. A phylogenetic tree was constructed with the default parameters and the aLRT statistical test model using PhyML [34]. Finally, the tree was rendered and displayed with TreeDyn [35], and the branches were annotated with 1000 resamplings for the bootstrap test values. The exon-intron organizations for all genes were obtained by using the online Gene Structure Display Server (GSDS, http://gsds.cbi.pku.edu.cn) with both the predicted coding sequences and the corresponding genomic sequences [36]. The ALOG protein sequences were statistically analyzed with the MEME program (http://meme-suite.org/) to confirm the presence of the conserved motifs using the following 
parameters: number of repetitions set as "any," maximum number of motifs of 5 , and optimum motif width set to $>5$ and $<150$ [37].

2.4. Microarray-Based Expression Analysis. All expression profile data, including those for the ALOG domain genes of maize and OsG1/G1Ls, which cover 60 tissues of B73 and 33 tissues of two indica rice varieties Zhenshan 97 (ZS97) and Minghui 63 (MH63), were collected from the PLEXdb (http://www.plexdb.org/) and CREP (http://crep.ncpgr.cn) databases [38, 39]. Then, the massively parallel signature sequencing (MPSS) data were used to determine the expression profiles of the genes with conflicting probe set signals [40]. All Arabidopsis microarray data were downloaded from the Gene Expression Omnibus database (http://www.ncbi .nlm.nih.gov/geo) using the GSE series accession numbers GSE5629, GSE5630, GSE5631, GSE5632, GSE5633, and GSE5634. The subsequent analysis of the gene expression data was performed in the statistical computing language $\mathrm{R}$ (http://www.r-project.org) using packages available from the Bioconductor project (http://www.bioconductor.org), and the raw data were processed with the Affymetrix Microarray Analysis Suite (MAS version 5, Affymetrix) [41]. The Euclidean distances of all OsG1/G1Ls were calculated, and the hierarchical method "complete linkage clustering" was applied to construct the clustering tree, which was displayed using the $\mathrm{R}$ package. The expression patterns of the OsG1/G1Ls were estimated and classified according to the hierarchical clustering results.

2.5. Coexpression Analysis with the MADS-Box Gene Family in Rice. All known MADS-box genes were obtained from the TIGR database via a putative function inquiry using the keyword "MADS." All expression profile data, including those for all of the MADS-box genes, which cover 33 ZS97 and MH63 tissues, were collected from the CREP (http:// crep.ncpgr.cn) database. The $\mathrm{R}$ language was used to calculate Pearson's correlation coefficients for OsG1/G1Ls and MADS-box genes, and the "as.dist" function was used to calculate the Euclidean distance matrix. The hierarchical clustering tree of coexpression was constructed with the "hclust" function using the Euclidean distance matrix. The coexpression network was finally constructed by igraph software with a cut-off value of 0.5 .

2.6. Detection of Expression Profiles of the OsG1/G1Ls in Rice. The growth of ZS97 was maintained under routine field management in Wuhan $\left(30^{\circ} 52^{\prime} \mathrm{N}, 114^{\circ} 32^{\prime} \mathrm{E}\right)$, China. To be consistent with the samples used in the previous microarray data (see Supplementary Table S1), the samples were harvested from ZS97 plants at the corresponding stages, including seedlings (three-leaf stage, Z12), young shoots (seedlings with two tillers, Z13), young roots (seedlings with 2 tillers, Z14), mature sheaths (secondary branch primordium differentiation stage, Z17), young flag leaves (5 days before heading, Z19), old flag leaves (14 days after heading, Z20), young panicles (4-5 cm, Z24), old panicles (heading stage, Z25), young stems (5 days before heading, Z26), spikelets (3 days after pollination, Z29), and endosperms (7 days after pollination, Z31). Calli (15 days after induction, Z4) were obtained after callus induction using ZS97 seeds. Total RNA was isolated from each sample with the TRIzol reagent (Invitrogen, Carlsbad, CA, USA), according to the manufacturer's instructions. The total RNA was treated with RNase-free DNase I (New England Biolabs, Ipswich, MA, USA) to eliminate genomic DNA contamination. Quantitative real-time PCR (qRT-PCR) was performed with the same procedures as previously reported [11]. The primers used in the present study are listed in Supplementary Table S2.

\section{Results}

3.1. Identification of ALOG Domain Genes. To identify ALOG domain genes in rice (Oryza sativa) and six other species, including Physcomitrella patens, Brachypodium distachyon, Zea mays, Sorghum bicolor, Arabidopsis thaliana, and Populus trichocarpa, a combined HMMER and BLAST search was performed on a local database as described in Materials and Methods. We finally identify a total of eightyfour sequences containing the ALOG domain from these seven species (see Supplementary Table S3). Specifically, only four ALOG domain genes are identified in Physcomitrella patens, while maize possesses nineteen ALOG domain genes in the present study (see Supplementary Table S3). A total of fourteen ALOG domain genes are identified in rice, among which four genes are novel ALOG domain genes compared with previous studies, which are sequentially named OsG1L10 to OsG1L13 in the present study [20, 23]. Furthermore, six of the fourteen OsG1/G1Ls have fulllength cDNA in KOME, and only OsG1L13 is absent in the UniProt annotation result (see Supplementary Table S4). Additionally, probe sets of 11 OsG1/G1Ls are found in the CREP database, supporting the subsequent expression analysis of ALOG domain genes in rice. Moreover, the number of amino acids of 13 OsG1/G1L proteins varies from 180 to 284, except for OsG1L12, with the shortest protein length of only 102 amino acids.

3.2. Chromosomal Distribution and Duplication Events of the ALOG Domain Genes in Rice. To show the chromosome location maps of the fourteen $O s G 1 / G 1 L s$, the 12 rice chromosomes were drawn as the chromosome pseudomolecules available in TIGR. The result shows that OsG1/G1Ls are unevenly distributed among eight of the twelve rice chromosomes (Figure 1). Generally, ten OsG1/G1Ls are located on 4 chromosomes, including two genes on Chr. 1 (OsG1L7 and OsG1L10), three genes on Chr. 2 (OsG1L1, OsG1L3, and OsG1L6), three genes on Chr. 5 (OsG1L8, OsG1L9, and OsG1L11), and two genes on Chr. 8 (OsG1L12 and OsG1L13). By contrast, the other four OsG1/G1Ls, OsG1L4, OsG1L2, OsG1, and OsG1L5, are evenly located on Chr. 4, Chr. 6, Chr. 7, and Chr. 10, respectively. In addition, the OsG1/G1Ls identified on Chr. 1, Chr. 5, and Chr. 8 tend to locate on the same chromosome arm within each chromosome. However, the OsG1/G1Ls on Chr. 2 are randomly allocated. 


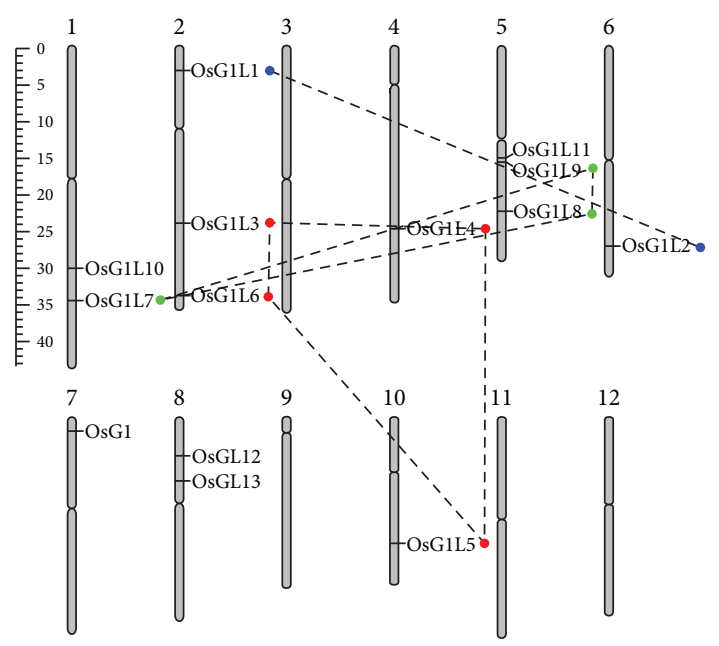

Figure 1: Genomic location and segmental genome duplication of the OsG1/G1Ls. The chromosome numbers are indicated at the top of each chromosome, and the genes present on the duplicated chromosomal segments of the genome are connected by lines. The scale on the left is in megabases (MB). The segmental duplicated genes were elucidated from PGDD with a parameter of $500 \mathrm{~kb}$.

Segmental duplication and tandem duplication play important roles in generating the members of a gene family during evolution [42]. In the present study, three groups of OsG1/G1Ls are involved in segmental duplication events. In the first group, OsG1L3, OsG1L6, OsG1L4, and OsG1L5 consist of four putative duplicated gene pairs, including OsG1L3/OsG1L4, OsG1L3/OsG1L6, OsG1L4/OsG1L5, and OsG1L5/OsG1L6. The second group contains three duplicated gene pairs formed by any two genes of OsG1L7, OsG1L8, and OsG1L9. The third group contains only one duplicated gene pair of OsG1L1/OsG1L2 (Figure 1). However, no tandem duplication is identified among the OsG1/ G1Ls. To assess the duplication time of the segmentally duplicated OsG1/G1Ls, the synonymous (Ks) and nonsynonymous substitutions $(\mathrm{Ka})$, and the $\mathrm{Ka} / \mathrm{Ks}$ ratios have been obtained from PGDD. The Ka/Ks ratios of each segmental duplicated gene pair range from 0.28 to 0.79 , with an average of 0.53 (smaller than 1), implying that the OsG1/G1Ls underwent relatively strong purifying selection. The approximate dates of the segmental duplication events were calculated from Ks, and all segmental duplications of the OsG1/G1Ls occurred 22.04-41.12 million years ago (see Supplementary Table S5). Further analysis of the duplication events in the seven species revealed that no duplication arose between dicots and monocots, and 54 of 57 (94.7\%) duplication events occurred no more than 150 million years ago (see Supplementary Table S5). These results suggested that the expansion of the ALOG gene family mainly occurred after the divergence of the monocotyledonous and dicotyledonous plants approximately 200 million years ago [43].

3.3. Phylogenetic and Structure Analysis of ALOG Domain Genes. To examine the evolutionary relationships among the OsG1/G1Ls in rice, an unrooted phylogenetic tree was constructed by PhyML (for details, see Materials and Methods). The 14 OsG1/G1Ls are classified into two distinct clusters with three subclasses named cluster I, cluster IIA, and cluster IIB (Figure 2(a)). Interestingly, all four newly identified members of the rice ALOG domain gene family, OsG1L10, OsG1L11, OsG1L12, and OsG1L13, are included in cluster I. Furthermore, the three genes with known functions, OsG1, OsG1L5, and OsG1L6, are all included in cluster II; however, OsG1L5 and OsG1L6 are in the same subclass of cluster IIA, whereas $O s G 1$ is assigned to the other subclass of cluster IIB. The phylogenetic data also indicate that OsG1L7, $O s G 1 L 8$, and OsG1L9 are paralogs of OsG1 (cluster IIB) and that OsG1L3, OsG1L4, and OsG1L5 are paralogs of OsG1L6 (cluster IIA). Moreover, OsG1 and OsG1L6 are also paralogous genes, which share a similar function in regulating the development of sterile lemma and lemma/palea, respectively $[20,21]$. In addition, comparison of the gene structures revealed that all the four genes in cluster I do not have UTRs and that none of the genes in cluster IIB contain introns, but almost all the genes in cluster IIA possess both introns and UTRs (Figure 2(b)). These data demonstrated that the three phylogenetically classified groups in rice share similar gene structures within each group.

To better understand the evolutionary relationships of the ALOG domain genes among the seven species, phylogenetic analysis of all ALOG proteins from the seven species was performed (Figure 3). The phylogenetic tree divides the ALOG domain genes into six distinct clusters (clusters A to F). The three groups of segmental duplicated genes of OsG1/G1Ls are assigned to clusters A, C, and E. OsG1, $B d G 1, S b G 1$, and $Z m G 1$ are orthologous genes in cluster $B$, which shows a similar classification with the previously reported G1 clade [20]. Moreover, GRMZM2G050917 is identified as another ortholog of OsG1 in maize, indicating that these four orthologs of $O s G 1$ might have the same function as OsG1. Similarly, the data have revealed four orthologs in the other three species for OsG1L5 (Bradi3g28800, Sb01g019290, GRMZM2G081515, and GRMZM2G034385) and OsG1L6 (GRMZM2G027302, GRMZM2G168371, Sb04g036620, and Bradi3g54800). Interestingly, the ALOG domain genes in clusters $\mathrm{B}, \mathrm{C}$, and $\mathrm{E}$ are all from monocotyledons, and the ALOG domain genes in clusters $\mathrm{D}$ and $\mathrm{F}$ are all from dicotyledons (Figure 3), suggesting that ALOG domain genes are evolutionarily conserved within monocotyledons or dicotyledons. Although cluster A contains the four genes of $P$. patens, the other thirteen genes are all from monocotyledons, indicating a close evolution of $P$. patens genes with monocotyledons. Taken together, these results provide evidence for the functional research of the uncharacterized but evolutionarily conserved genes.

To gain further insights into the structural diversity of ALOG domain genes, we first compared the exon/intron structure in the coding sequences of ALOG genes (see Supplementary Figure S1). Most of the genes from the dicotyledonous plants seem to lack introns, while the genes from the monocotyledons display more complicated gene structures, and most of them contain introns. Notably, all genes from $B$. distachyon do not contain any intron. However, no uniform structural pattern was observed for the genes in $P$. patens. Thereafter, the MEME motif search tool was used to investigate the conserved motifs of the 


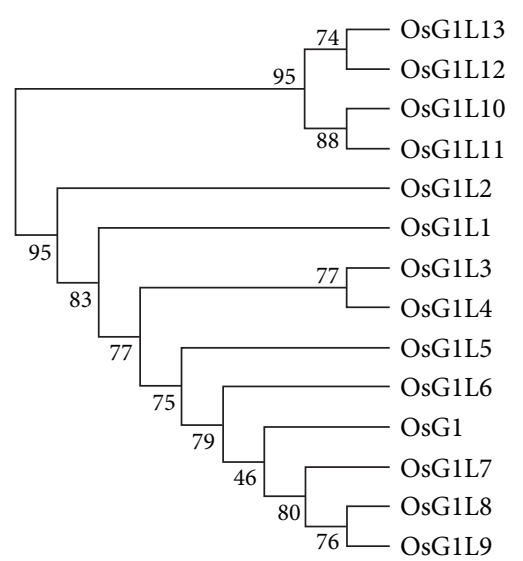

(a)

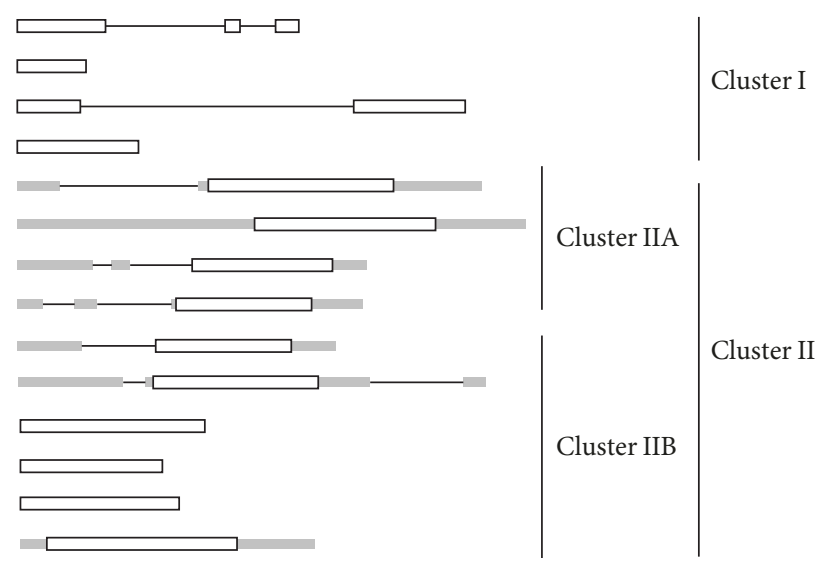

(b)

Figure 2: Phylogenetic relationships of the OsG1/G1L proteins and the exon-intron organizations of the corresponding genes. (a) The unrooted tree was generated with the default parameters and the "aLRT" statistical test model using PhyML. (b) Using the sequences from the TIGR locus in Table S4, the exons, introns, and UTRs are represented by white boxes, lines, and black boxes, respectively.

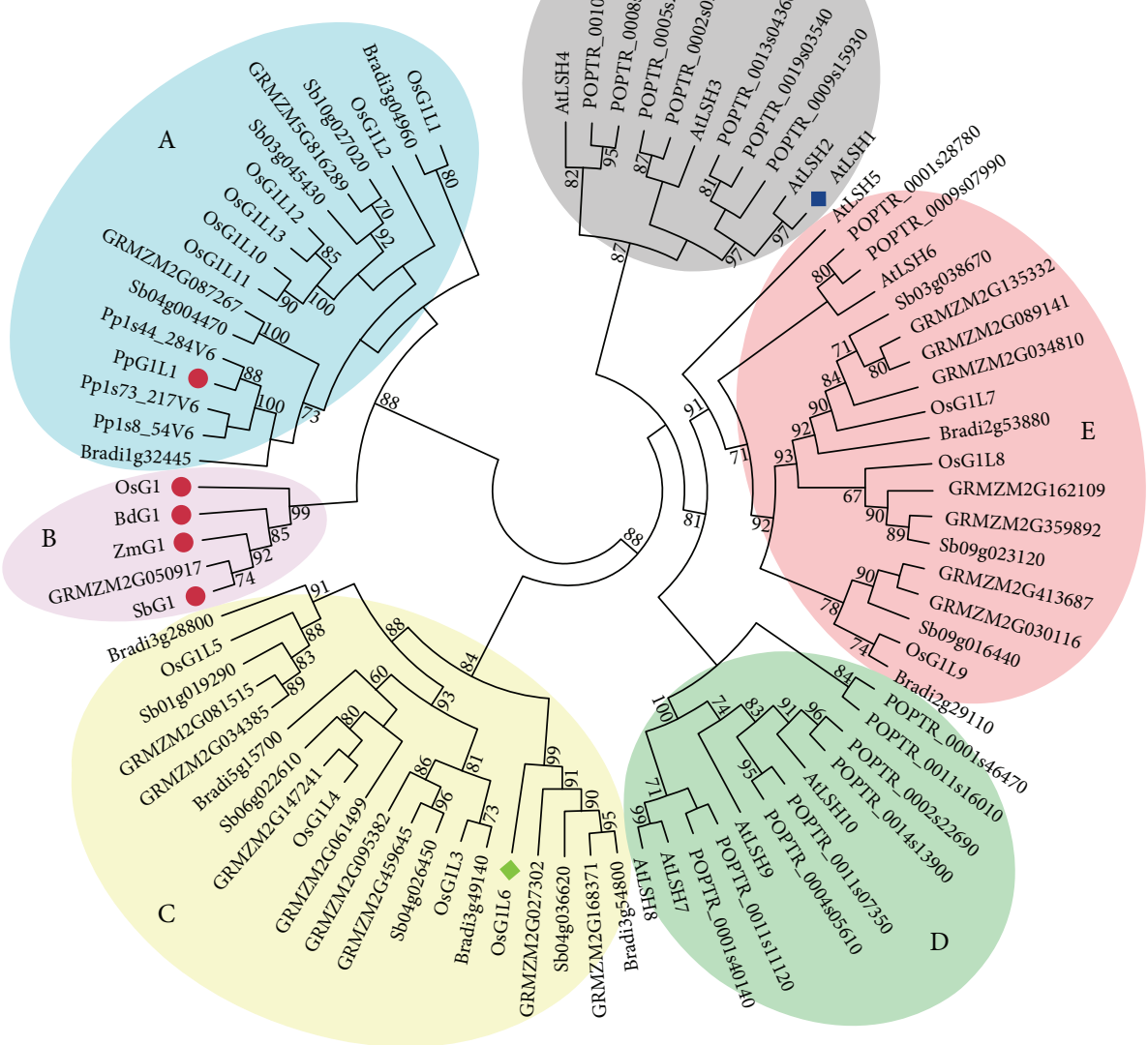

FIgURE 3: Phylogenetic relationships of the ALOG domain proteins in rice and six other species. Subgroups A to F are indicated in different colored ovals.

ALOG protein sequences. We identify five distinct conserved motifs among the ALOG proteins from rice and the other six species (see Supplementary Figure S2). Motifs 1, 2, and 3 specify the ALOG domain, and motifs 4 and 5 are newly discovered in the present study (see Supplementary Table S6). Motifs 1, 2, and 3 are observed in almost all members of the ALOG domain gene family in the seven species, with the exception of the four newly discovered rice 
genes (OsG1L10, OsG1L11, OsG1L12, and OsG1L13) and two maize genes (GRMZM2G050917 and GRMZM2G089141), which lack one or two of the three motifs. This result suggested that motifs 1,2 , and 3 are highly conserved among the seven species. Significantly, although the function of motifs 4 and 5 is unknown, these two motifs are only present in monocotyledonous proteins from cluster C, indicating that motifs 4 and 5 might have specific functions in monocots.

3.4. The Tissue-Specific Expression Patterns of OsG1/G1Ls Revealed the Potential Biological Functions. To explore the expression patterns and potential functions of the OsG1/ G1Ls, we obtained the microarray data for 11 OsG1/G1Ls in 33 tissues from two cultivated varieties (ZS97 and MH63), which covered almost the entire life cycle of rice. A hierarchical clustering analysis based on the signal values showed that the expression profiles of OsG1/G1Ls can be classified into two major groups of $\mathrm{A}$ and $\mathrm{B}$ and further subdivided into four distinct clusters of AI, AII, BI, and BII (Figure 4(a)). OsG1 and OsG1L6 exhibit uniquely high expression levels in the panicles, which is spatially consistent with their function in regulating the development of floral organs and panicles [20-22, 25, 44]. Furthermore, OsG1L6 also displays high expression in the plumules, under both dark and light conditions, implying that OsG1L6 has other functions besides regulating panicle development. In fact, a previous study has already revealed the pleiotropic effect of OsG1L6 in regulating plant height, floral development, and grain yield in rice [24]. The present data also showed that the newly discovered OsG1L10 and OsG1L11 genes belonging to cluster AI are expressed at extremely low levels in almost all studied tissues. In contrast, OsG1L2 exhibits remarkably high expression levels in almost every tissue. These results indicated that OsG1L10 and OsG1L11 might have lost their functions, whereas OsG1L2 plays an important role throughout the development of rice and likely represents a housekeeping gene in rice. In addition, OsG1L4 in cluster BI is highly expressed in seedlings, young shoot, and young stem, while OsG1L7 in the same cluster has a relatively high expression level in mature sheath and stem (Figure 4(a)). Taken together, these results suggested that the OsG1/G1Ls have evolved specific biological functions in diverse tissues throughout the rice life cycle.

To further confirm the tissue-specific expression patterns of the OsG1/G1Ls, the relative expression levels of 9 OsG1/G1Ls covering the four clusters AI, AII, BI, and BII were investigated in 12 tissues by qRT-PCR (Figure 4(b)). The expression patterns demonstrated several interesting results. First, six genes, including OsG1L1, OsG1L2, OsG1L3, OsG1L6, OsG1L7, and OsG1L13, exhibit high expression levels in mature sheaths (Z17), revealing a potential role for these genes in regulating plant height or the physiological function of rice sheath. Second, the remaining three genes, OsG1L5, OsG1L8, and OsG1L9, are highly expressed in young roots (Z14), and OsG1L5 also shows high expression levels in calli (Z4). Since OsG1L5/TAWAWA1 was formerly revealed to regulate meristem activity in rice shoot apex [28], the expression of OsG1L5 in young roots and calli can be explained by the fact that the root apex and calli also contain an abundance of meristems. However, the absence of OsG1L8 and OsG1L9 in calli (Z4) suggested that these genes might have a different molecular function than OsG1L5. Moreover, the expression patterns of OsG1L8 and OsG1L9 revealed by qRT-PCR are highly similar to those revealed by microarray, particularly the relatively high expression levels in Z14, Z17, Z26, and Z29 of both datasets (Figures 4(a) and 4(b)), which experimentally confirmed the reliability of the microarray data. Third, when we compared the expression of OsG1/G1Ls between young (Z24) and old (Z25) panicles, most genes show similar expression levels. For instance, OsG1L1 shows similar high expression levels, and OsG1L2 exhibits equivalent low expression levels, while OsG1L7, OsG1L8, OsG1L9, and OsG1L13 are simultaneously absent in these two tissues. However, OsG1L3, OsG1L5, and OsG1L6 show higher expression levels in young panicle than in old panicle. These data were also supported by the fact that both OsG1L5 and OsG1L6 primarily function in young panicles to regulate the development of inflorescence and spikelet $[21,23,25,28]$; thus, their expression levels can hardly be detected in old panicles.

3.5. Coexpression Analysis Reveals the Underlying Relationships between OsG1/G1Ls and MADS-Box Genes in Rice. The previously reported OsG1/G1Ls mainly participate in the developmental regulation of floral organs in rice [20, 21, 23-25, 28]. It is well known that the identities of the floral organs in plants are controlled by the combined activities of MADS-box genes. To reveal the underlying regulatory relationships of OsG1/G1Ls and MADS-box genes, we constructed a hierarchical clustering tree and a network of coexpression by using the microarray data of OsG1/ G1Ls and MADS-box genes in rice. The clustering results revealed that $O s G 1$ is classified into the same cluster with OsMADS32, OsMADS65, OsMADS98, OsMADS15, OsMADS34, OsMADS17, OsMADS1, OsMADS6, and OsMADS5 (Figure 5(a)). Expectedly, OsMADS1, OsMADS17, and OsMADS34 are in the same cluster because OsMADS17 is a direct target gene of OsMADS1, and OsMADS1 can negatively regulate the expression of OsMADS34 in young spikelets [45, 46]. Coincidentally, the lodicules and stamens are converted to multiple lemma/glume-like organs in the OsMADS1 mutant, which shows almost the same phenotype as the OsG1 mutant [20,45]. These results strongly suggested that OsMADS1 and OsG1, perhaps as well as other genes in this cluster, function in the same molecular pathway to specify floral organ identity. Additionally, stem elongation in the double mutant of OsMADS22 and OsMADS55 is markedly reduced [47], and OsG1L7 is highly coexpressed with these two genes (Figure 5(a)). Moreover, OsG1L7 shows a relatively high expression level in the sheath and stem (Figures 4(a) and 4(b)), suggesting that OsG1L7 functions synergistically with OsMADS22 and OsMADS55 to regulate stem elongation.

Subsequently, the coexpression network between OsG1/ G1Ls and MADS-box genes was constructed by using igraph (Figure 5(b)). The network shows complicated relationships among different MADS-box genes; however, a much simpler 


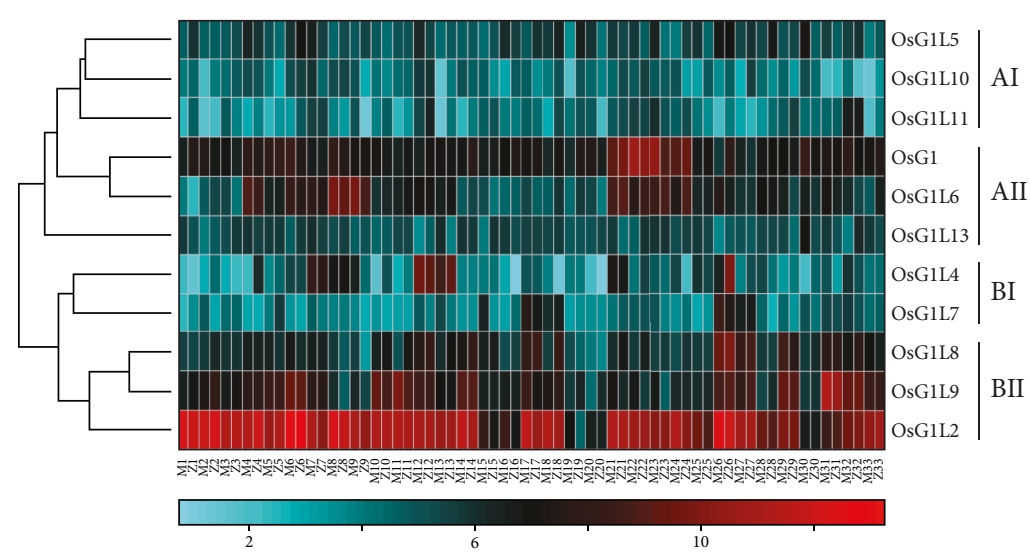

(a)
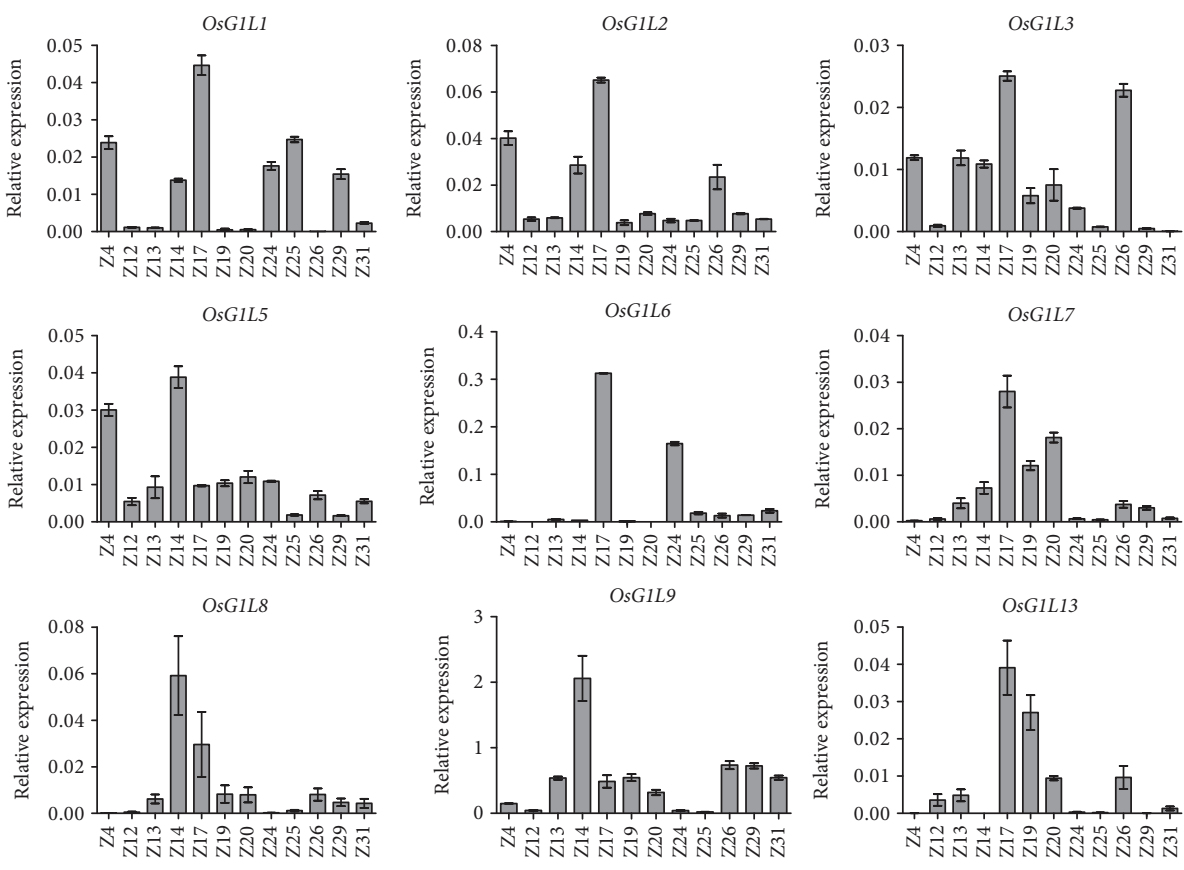

Z4: calliat 15 days after
induction
Z12: seedlings at the three-
leaf stage
Z13: shoots of seedlings
with two tillers
Z14: roots of seedlings
with two tillers
Z17: sheaths at the secondary
branch primordium
differentiation stage
Z19: flag leaves at 5 days
before heading

Z20: flag leaves at 14 days
after heading
Z24: panicles at $4-5 \mathrm{~cm}$
length
Z25: panicles at the headin
stage
Z26: stems at 5 days before
heading
Z29: spikeletsat 3 days after
pollination
Z31: endosperms at 7 days
after pollination

(b)

FIGURE 4: Genome-wide expression profiles and qRT-PCR analysis of OsG1/G1Ls. (a) The hierarchical clustering displays the expression profiles of the 11 OsG1/G1Ls using the matching probes in the Affymetrix microarray. The color scale at the left bottom represents the $\log _{2}$-transformed expression values. Cyan indicates a low expression level, black indicates a medium expression level, and red indicates a high expression level. The thirty-three samples of MH63 and ZS97 were sequentially marked as M1 to M33 and Z1 to Z33 on the $x$-axis, respectively. The detailed sample information was listed in Supplementary Table S1. (b) qRT-PCR analysis of 9 OsG1/G1Ls. The twelve analyzed samples were indicated as Z4 to Z31 on the $x$-axis because they were sampled according to the tissues and developmental stages in the microarray (see Supplementary Table S1). The values on the $y$-axis represent the relative expression levels. The error bars represent the standard deviation of the means of three independent replicates.

network is detected for OsG1/G1Ls. For example, OsG1L4, OsG1L7, OsG1L8, OsG1L9, OsG1L11, and OsG1L6 show interaction networks with only one to three MADS-box genes. However, OsG1 was found to play a pivotal role in integrating the interaction between OsMADS50 and a network composed of many other MADS-box genes, including 


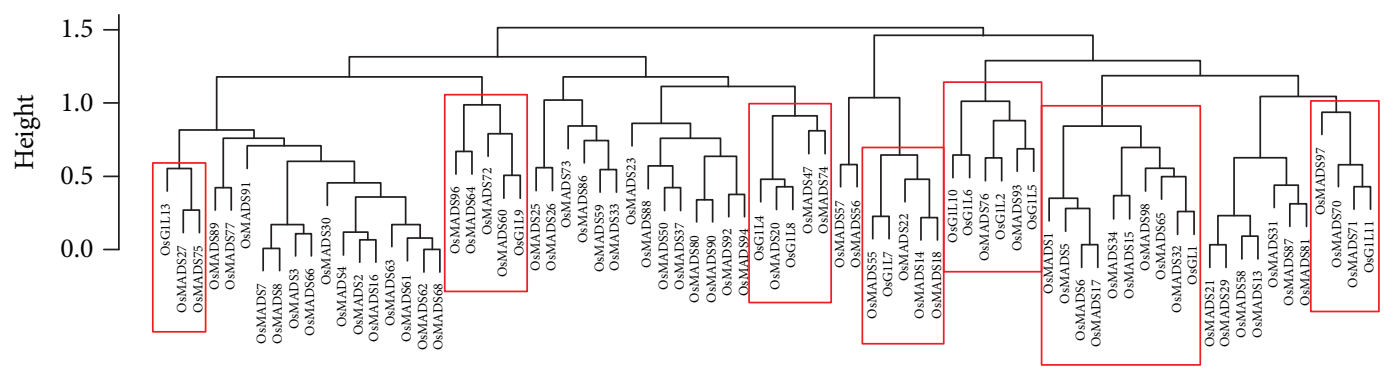

(a)

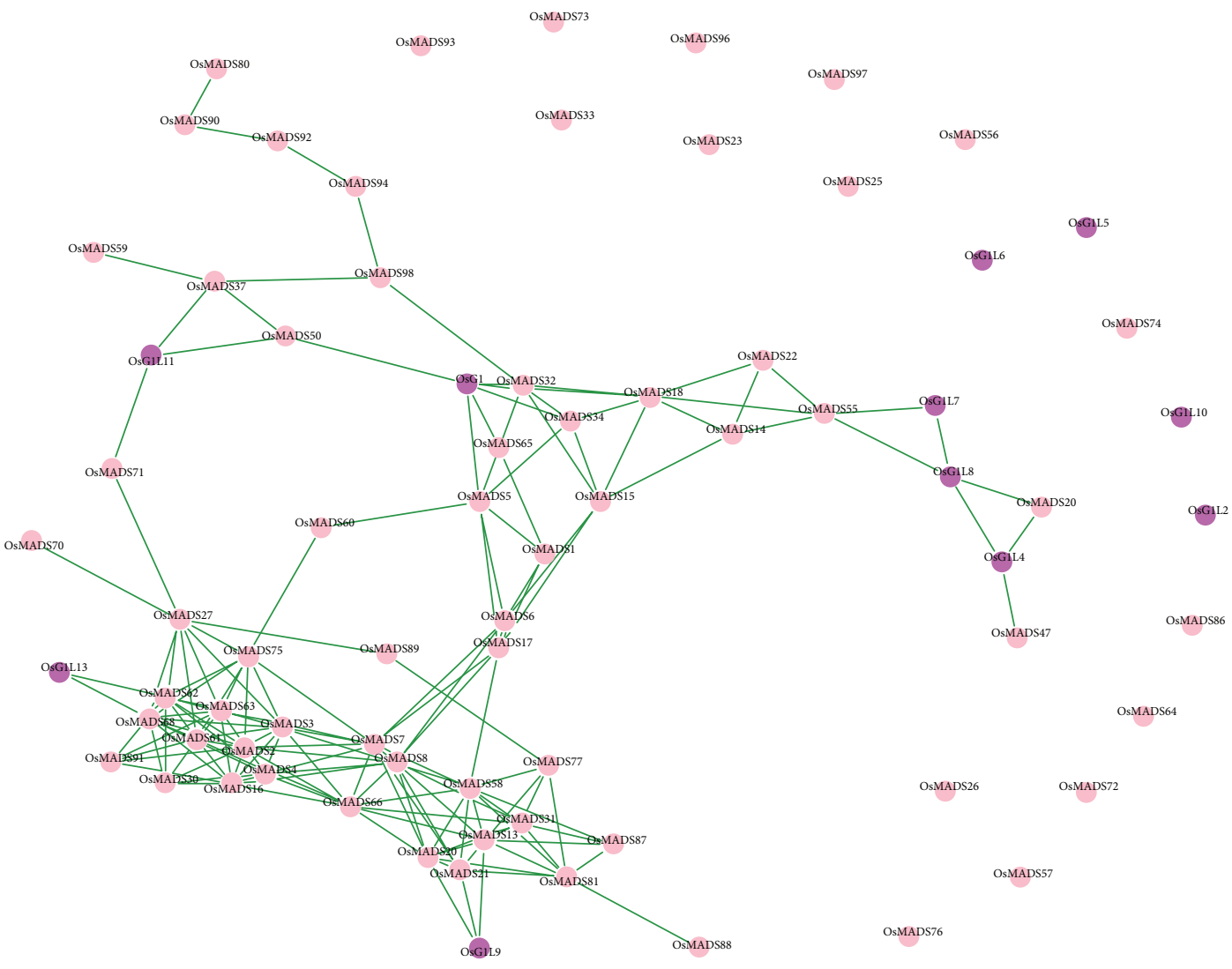

(b)

FIGURE 5: The hierarchical clustering tree and coexpression network between OsG1/G1Ls and MADS-box genes in rice. (a) The hierarchical cluster dendrogram showing coexpression modules. The red boxes represent the analyzed coexpression subgroups. The hierarchical clustering tree of coexpression was constructed with the hclust function in R. (b) The gene coexpression network between OsG1/G1Ls and OsMADS genes. The green lines between two genes represent the coexpression relationships, and their length represents the coexpression signal intensity. The coexpression network was drawn by igraph software with a cut-off value of 0.5 .

OsMADS32, OsMADS18, OsMADS34, OsMADS65, OsMADS5, OsMADS14, OsMADS15, OsMADS1, OsMADS6, and OsMADS17 (Figure 5(b)). Interestingly, OsMADS50 is an important flowering activator considered to be an upstream regulator of OsMADS1, OsMADS14, OsMADS15, and OsMADS18 [48]. Taken together, this coexpression network further indicated that OsG1 functions between OsMADS50 and the downstream MADS-box genes, such as OsMADS1, to regulate the development of inflorescence in rice. However, the authentic relationships between the OsG1/G1Ls and MADS-box genes need more molecular and genetic evidence, which could be accomplished in future studies.
3.6. Comparative Analysis of the Expression Patterns of ALOG Domain Genes in Arabidopsis and Maize. To investigate the potential roles of the ALOG domain genes in Arabidopsis and maize, we determined the expression patterns of all ALOG domain genes in these two species by using the available microarray data. The ALOG domain genes in Arabidopsis are primarily divided into two major groups (I and II) based on the hierarchical clustering analysis (Figure 6). The first group (I) is represented by AtLSH1, AtLSH3, AtLSH4, and AtLSH10, which show high expression levels in the hypocotyl (A8), 1st node (A9), 2nd internode (A10), and shoot apex (A37-A39). These data suggested that these 


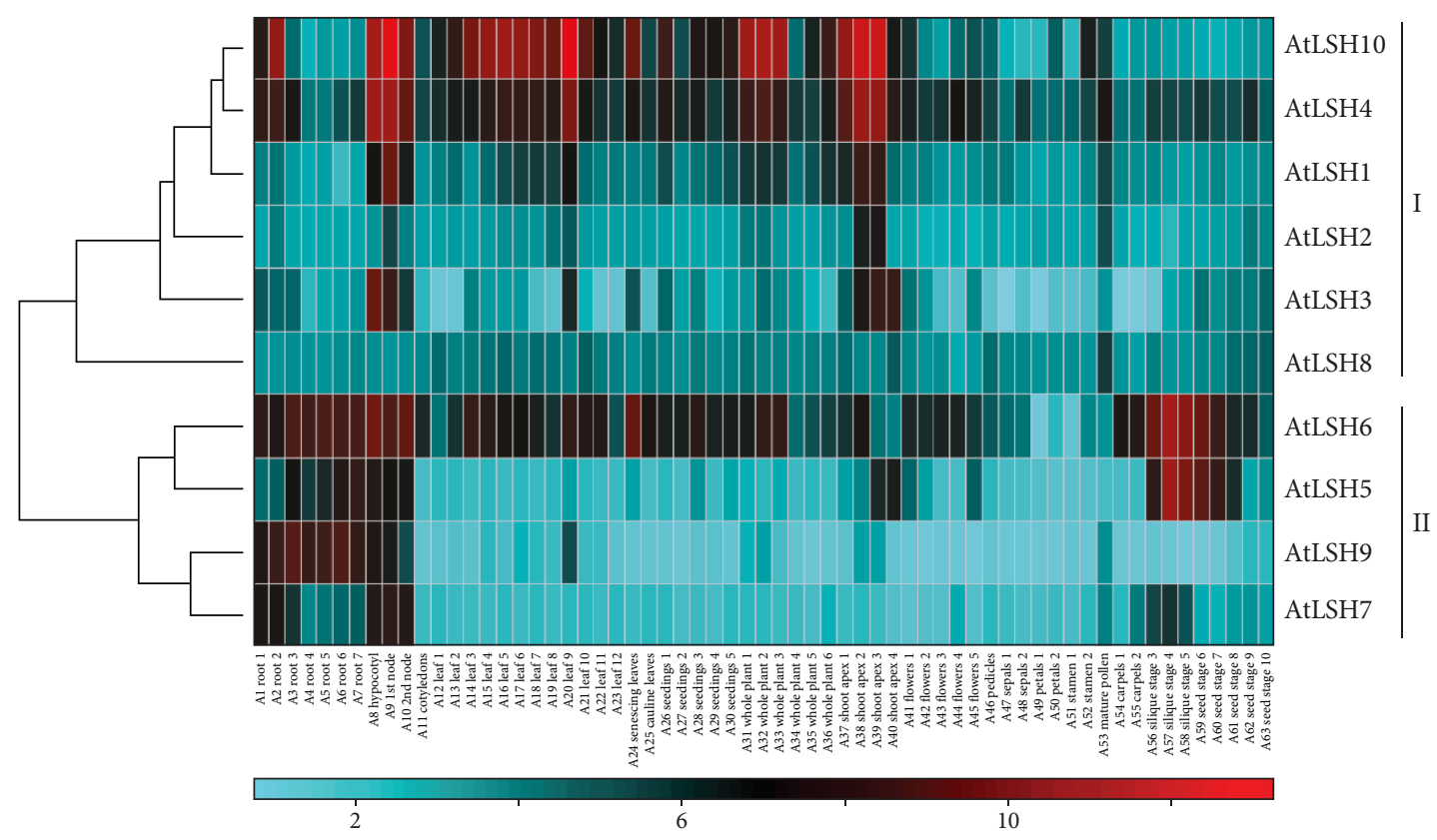

Figure 6: Genome-wide expression profile of ALOG domain genes in Arabidopsis. The hierarchical clustering displays the expression profiles of the 10 ALOG domain genes (AtLSH1 to AtLSH10). The color scale at the top left represents the $\log _{2}$-transformed expression values. Cyan indicates a low expression level, black indicates a medium expression level, and red indicates a high expression level.

genes regulate the development of the hypocotyl and shoot apex. Indeed, AtLSH1 mediates a light-dependent regulation of hypocotyl development [26]; AtLSH3 and AtLSH4 are important for the maintenance of shoot apical meristem and floral organ development $[49,50]$. Furthermore, AtLSH4 and AtLSH10 display a strong coexpression pattern and are highly expressed almost throughout the Arabidopsis life cycle, suggesting that both genes are functionally important in most of the developmental stages. However, AtLSH2 exhibits a similar expression pattern with AtLSH1 but is expressed only in the shoot apex (A38 and A39), suggesting a more specified function of AtLSH2 in the shoot apex. The second group (II) is composed of AtLSH5, AtLSH6, AtLSH7, and AtLSH9, which display high expression levels in the root, hypocotyl, and node (A1-A10). This expression pattern is consistent with the revealed role of AtLSH9 in the regulation of the hypocotyl length [51] and indicates that AtLSH5, AtLSH6, AtLSH7, and AtLSH9 might have a similar function in hypocotyls as well as unknown functions in roots. Notably, AtLSH5, AtLSH6, and AtLSH7 might also function in the development of siliques since these genes all show obviously high expression levels in siliques (A56-A58), except for AtLSH9.

On the other hand, we found that the ALOG domain genes in maize also exhibit tissue-specific expression patterns that were similar to those in rice and Arabidopsis (see Supplementary Figure S3). For instance, GRMZM2G459645, GRMZM2G095382, GRMZM2G081515, GRMZM2G147241, GRMZM2G061499, and GRMZM2G034385 are highly expressed in the stem and shoot apical meristem (SAM), resembling the highly expressed AtLSH1, AtLSH3, AtLSH4, and AtLSH10 in Arabidopsis hypocotyl, node, and shoot apex. Furthermore, GRMZM2G162109, GRMZM2G168371, and
GRMZM2G027302 are not only expressed in the stem and SAM but also highly expressed in the husk and seeds. Specifically, GRMZM2G168371 and GRMZM2G027302, the maize orthologs of rice OsG1L6, exhibit robustly high expression levels in maize husk and seeds, suggesting that these two maize orthologs might have the same function as OsG1L6. This result demonstrated a conserved role of ALOG domain genes in regulating the development of inflorescence in maize and rice. However, the orthologous gene of OsG1 in maize, GRMZM2G050917, shows extremely low expression levels in almost all examined tissues, which implies that this ortholog may have lost its molecular function in maize. Taken together, a comparative analysis of tissue-specific expression patterns of ALOG domain genes has provided an alternative strategy to reveal their conserved biological functions among species.

\section{Discussion}

In the present study, we conducted a comprehensive bioinformatics analysis of ALOG domain genes in rice and six other species. A total of eighty-four ALOG domain genes are identified, among which fourteen OsG1/G1Ls are found to be unevenly distributed on eight of the twelve chromosomes. Phylogenetic and structural analysis showed that ALOG domain genes are evolutionarily conserved within monocotyledons or dicotyledons and that the ALOG domain-specified motifs 1,2 , and 3 are highly conserved among different species. However, the newly identified motifs 4 and 5 are only present in monocots, presenting a specific function of these two motifs in monocots. The expression analysis showed that OsG1/G1Ls possess specific biological functions in diverse tissues throughout the rice life 
cycle. The coexpression results revealed that OsMADS1 and OsG1 function in the same molecular pathway to specify floral organ identity; OsG1L7 is potentially associated with OsMADS22 and OsMADS55 to regulate stem elongation. Moreover, the coexpression network showed that OsG1 integrates OsMADS50 and the downstream MADS-box genes to regulate the development of rice inflorescence. Finally, the comparative expression analysis revealed the conserved biological functions of ALOG family genes among rice, maize, and Arabidopsis. These results have shed light on the functional study of ALOG family genes, which will further assist plant breeders for selecting candidate genes during the breeding of rice and other crops.

The present data suggested that segmental duplications mainly contributed to the expansion of OsG1/G1Ls, which occurred 22.04-41.12 million years ago. However, the average $\mathrm{Ka} / \mathrm{Ks}$ ratio of the segmental duplicated gene pairs is 0.53 , indicating that OsG1/G1Ls are under purifying selection. Moreover, all $\mathrm{Ka} / \mathrm{Ks}$ ratios of the duplicated genes within the seven species are less than 1, implying that the ALOG family genes are undergoing strong purifying selection (see Supplementary Table S5). In natural selection, purifying selection or negative selection involves the selective removal of alleles that are deleterious, which could also be applied in artificial selection to preserve preferred traits and remove unwanted traits according to human needs. As previous studies reported, the mutation of ALOG family genes is typically associated with unwanted traits, such as abnormal phenotype of hulls in the rice mutant of OsG1 and TH1/OsG1L6 $[20,21,25]$ and the single-flower primary inflorescence in the tomato mutant of the TERMINATING FLOWER gene, which is an ALOG domain gene identified from tomato [52]. These results can partially explain why the ALOG family genes are under purifying selection and suggest that the reserved ALOG family genes play crucial roles during plant development.

The phylogenetic analysis has divided the ALOG family genes into six clusters, in which monocotyledonous ALOG genes constitute clusters $\mathrm{A}, \mathrm{B}, \mathrm{C}$, and $\mathrm{E}$, while dicotyledonous ALOG genes are all classified into clusters D and F (Figure 3). The phylogenetic classification seems to be consistent with the gene structure and protein motif distribution. For example, the ALOG family genes of monocotyledonous plants generally contain a number of introns, whereas dicotyledonous plants have fewer introns than the monocots (see Supplementary Figure S1). Furthermore, all of the ALOG family proteins of the dicotyledons do not have putative motifs 4 and 5, which are identified only in monocotyledons (see Supplementary Figure S2). These data not only suggested that the ALOG family genes are evolutionarily and structurally conserved within monocotyledons or dicotyledons but also implied that the ALOG family genes in dicotyledons preferentially lose introns compared with monocotyledons during evolution. This observation was similar to a previous result showing that intron losses are roughly 12.6 and 9.8 times more frequent than intron gains in the recent evolution of Arabidopsis thaliana and Oryza sativa, respectively [53]. However, there is an exception that all 9 ALOG family genes in Brachypodium distachyon contain no introns (see Supplementary Figure S1), indicating that intron losses are occasionally species-specific events. To further reveal the underlying mechanism of structure variation, why and how the intron losses of ALOG family genes, including precise and imprecise intron losses [54], were fixed during evolution should be answered in the future.

OsG1/G1Ls are composed of fourteen members that were identified in the present study. Currently, several studies have revealed the functional roles of three members in OsG1/G1Ls, showing that $O s G 1$ specifies sterile lemma identity in rice spikelets [20], OsG1L5/TAWAWA1 regulates inflorescence development by modifying meristem activity [28], and OsG1L6/TH1/BH1/AFD1 pleiotropically affects plant height, grain size, and particularly the development of spikelets, lemmas, and paleae [21-25, 44]. Compared to the ALOG gene family in rice, the MADS-box gene family is a much more extended family containing as many as 75 MADS-box genes [55]. Numerous studies have confirmed the importance of MADS-box genes in floral organ specification, plant growth, and resistance to biotic or abiotic stresses [45-48, 56-58]. The overlapping functions of OsG1/G1Ls and MADS-box genes, particularly their effects on floral organ identity and meristem activity, have prompted us to investigate their relationships by coexpression analysis. The results showed that OsG1 probably functions between OsMADS50 and many other MADS-box genes, such as OsMADS32, OsMADS18, OsMADS34, OsMADS65, OsMADS5, OsMADS14, OsMADS15, OsMADS1, OsMADS6, and OsMADS17, to specify floral organ identity. Among these MADS-box genes, OsMADS32 regulates floral organ identity in rice, OsMADS1 plays an important role in rice floral organ identity specification and floral meristem determinacy $[45,46,59,60]$, OsMADS1 and OsMADS15 are both required to ensure sexual reproduction in rice [61], and OsMADS34 specifies the identities of floral organs $[62,63]$. Based on these results, we propose that OsG1 functions synergistically with or upstream of OsMADS1, OsMADS15, OsMADS32, and OsMADS34 genes to specify the identities of floral organs in rice.

Comparative expression analysis within or between species is an efficient method to reveal the potential function of candidate genes. In the present study, we compared the tissue-specific expression patterns of ALOG domain genes among rice, maize, and Arabidopsis and found that several ALOG domain genes from different species prefer to express in similar tissues. For example, AtLSH5, AtLSH6, AtLSH7, and AtLSH9 display high expression levels in the hypocotyl and node, which correspond to the high expression of OsG1L7 and OsG1L8 in rice sheath and stem (Figures 4(a) and 6). The high expression of GRMZM2G162109, GRMZM2G168371, and GRMZM2G027302 in maize husk and seeds resembles the markedly high expression of OsG1 and OsG1L6 in rice panicles (Figure 4(a) and Figure S3). The conserved tissue-specific expression patterns of ALOG family genes between species provided the opportunity to investigate their conserved functions. Recently, a high-resolution spatiotemporal transcriptome analysis was successfully applied to reveal the regulatory 
and structural gene networks across tissue and developmental spectra in tomato [64]. Moreover, a previous time-course transcriptome analysis revealed that the flowering pathway genes are expressed during the same time quantum at approximately 8:00 am, indicating that the temporal consistency of gene expression is necessary for genes in the same pathway [65]. Therefore, future dissection of gene functions or regulatory networks needs more precise expression data from a series of high-resolution spatiotemporal samples.

\section{Data Availability}

The data used to support the findings of this study are available from the corresponding author upon request.

\section{Conflicts of Interest}

The authors declare no conflicts of interest.

\section{Authors' Contributions}

$\mathrm{Na} \mathrm{Li}$ and Chuan Liu designed the study and wrote the manuscript. Na Li, Yang Wang, and Jing Lu performed the experiments and analyzed the data. Yang Wang and Chuan Liu revised the manuscript. $\mathrm{Na} \mathrm{Li}$ and Yang Wang are co-first authors. All authors read and approved the final manuscript.

\section{Acknowledgments}

The authors would like to thank Dr. Xinxin Peng, Dr. Yufang Pan, and Dr. Shengli Jing (College of Life Sciences, Wuhan University) for the suggestions on the statistical analysis. They would also like to thank Dr. Kai Guo (College of Life Science and Technology, Huazhong Agricultural University) for the insightful suggestions on the implementation of bioinformatics tools. This research was financially supported by grants from the China Postdoctoral Science Foundation (2017M622506).

\section{Supplementary Materials}

Figure S1: the gene structure of ALOG domain genes in seven species. The size of each gene can be estimated using the black bar (100 bp) at the bottom of the figure. White boxes, exons; gray boxes, UTRs; lines, introns. Figure S2: distribution of conserved motifs in ALOG domain proteins identified using the MEME search tool. Five putative motifs were identified, in which motifs 1, 2, and 3 specify the ALOG domain, while motifs 4 and 5 are newly discovered in the present study. Different motifs are indicated by different colors, and the names of all members are shown at the top of the figure. The motif sizes are indicated at the bottom of the figure. Figure S3: genome-wide expression profile of ALOG domain genes in maize. The hierarchical clustering displays the expression profiles of the 16 ALOG domain genes in maize. The color scale at the top left represents the $\log _{2}$-transformed expression values. Cyan indicates a low expression level, black indicates a medium expression level, and red indicates a high expression level. Table S1: tissue samples of different developmental stages throughout the life cycle of rice. Table S2: the qRT-PCR primers used in the present study. Table S3: the eighty-four ALOG domain genes identified from the seven species. Table S4: information and characteristics of the identified OsG1/G1Ls in the rice genome. Table S5: the segmental duplication events of the ALOG family genes. Table S6: the conserved motifs identified from ALOG family proteins. (Supplementary Materials)

\section{References}

[1] Y. Li, J. Xiao, L. Chen et al., "Rice functional genomics research: past decade and future," Molecular Plant, vol. 11, no. 3, pp. 359-380, 2018.

[2] Y. Han, Y. Cao, H. Jiang, and T. Ding, "Genome-wide dissection of the chalcone synthase gene family in Oryza sativa," Molecular Breeding, vol. 37, no. 10, 2017.

[3] J. Ma, Y. Yang, W. Luo et al., "Genome-wide identification and analysis of the MADS-box gene family in bread wheat (Triticum aestivum L.)," PLoS One, vol. 12, no. 7, article e0181443, 2017.

[4] Y. Piao, K. Jin, Y. He et al., "Genome-wide identification and role of MKK and MPK gene families in clubroot resistance of Brassica rapa," PLoS One, vol. 13, no. 2, article e0191015, 2018.

[5] J. Wang, Z. Wu, Z. Shen et al., "Genome-wide identification, phylogeny, and expression analysis of ARF genes involved in vegetative organs development in switchgrass," International Journal of Genomics, vol. 2018, Article ID 7658910, 13 pages, 2018.

[6] Y. Xu, F. Liu, G. Han, and B. Cheng, "Genome-wide identification and comparative analysis of phosphate starvationresponsive transcription factors in maize and three other gramineous plants," Plant Cell Reports, vol. 37, no. 5, pp. 711-726, 2018.

[7] W. Xue, Y. Xing, X. Weng et al., "Natural variation in Ghd7 is an important regulator of heading date and yield potential in rice," Nature Genetics, vol. 40, no. 6, pp. 761-767, 2008.

[8] W. H. Yan, P. Wang, H. X. Chen et al., "A major QTL, Ghd8, plays pleiotropic roles in regulating grain productivity, plant height, and heading date in rice," Molecular Plant, vol. 4, no. 2, pp. 319-330, 2011.

[9] B. H. Koo, S. C. Yoo, J. W. Park et al., "Natural variation in OsPRR37 regulates heading date and contributes to rice cultivation at a wide range of latitudes," Molecular Plant, vol. 6, no. 6, pp. 1877-1888, 2013.

[10] H. Gao, M. Jin, X. M. Zheng et al., "Days to heading 7, a major quantitative locus determining photoperiod sensitivity and regional adaptation in rice," Proceedings of the National Academy of Sciences of the United States of America, vol. 111, no. 46, pp. 16337-16342, 2014.

[11] C. Liu, G. Song, Y. Zhou et al., "OsPRR37 and Ghd7 are the major genes for general combining ability of DTH, PH and SPP in rice," Scientific Reports, vol. 5, no. 1, article 12803, 2015.

[12] X. J. Song, W. Huang, M. Shi, M. Z. Zhu, and H. X. Lin, “A QTL for rice grain width and weight encodes a previously unknown RING-type E3 ubiquitin ligase," Nature Genetics, vol. 39, no. 5, pp. 623-630, 2007.

[13] H. Mao, S. Sun, J. Yao et al., "Linking differential domain functions of the GS3 protein to natural variation of grain size in rice," Proceedings of the National Academy of Sciences of 
the United States of America, vol. 107, no. 45, pp. 1957919584, 2010.

[14] Y. Li, C. Fan, Y. Xing et al., "Natural variation in GS5 plays an important role in regulating grain size and yield in rice," Nature Genetics, vol. 43, no. 12, pp. 1266-1269, 2011.

[15] S. Wang, K. Wu, Q. Yuan et al., "Control of grain size, shape and quality by OsSPL16 in rice," Nature Genetics, vol. 44, no. 8, pp. 950-954, 2012.

[16] S. Wang, S. Li, Q. Liu et al., "The OsSPL16-GW7 regulatory module determines grain shape and simultaneously improves rice yield and grain quality," Nature Genetics, vol. 47, no. 8, pp. 949-954, 2015.

[17] D. Xia, H. Zhou, R. Liu et al., "GL3.3, a novel QTL encoding a GSK3/SHAGGY-like kinase, epistatically interacts with GS3 to produce extra-long grains in rice," Molecular Plant, vol. 11, no. 5, pp. 754-756, 2018.

[18] S. Sun, L. Wang, H. Mao et al., "A G-protein pathway determines grain size in rice," Nature Communications, vol. 9, no. 1 , p. $851,2018$.

[19] A. Shomura, T. Izawa, K. Ebana et al., "Deletion in a gene associated with grain size increased yields during rice domestication," Nature Genetics, vol. 40, no. 8, pp. 10231028, 2008.

[20] A. Yoshida, T. Suzaki, W. Tanaka, and H. Y. Hirano, "The homeotic gene long sterile lemma (G1) specifies sterile lemma identity in the rice spikelet," Proceedings of the National Academy of Sciences of the United States of America, vol. 106, no. 47, pp. 20103-20108, 2009.

[21] X. Li, L. Sun, L. Tan et al., “TH1, a DUF640 domain-like gene controls lemma and palea development in rice," Plant Molecular Biology, vol. 78, no. 4-5, pp. 351-359, 2012.

[22] X. Wei, X. Zhang, G. Shao et al., "Fine mapping of $B H 1$, a gene controlling lemma and palea development in rice," Plant Cell Reports, vol. 32, no. 9, pp. 1455-1463, 2013.

[23] D. Yan, Y. Zhou, S. Ye, L. J. Zeng, X. M. Zhang, and Z. H. He, "BEAK-SHAPED GRAIN 1/TRIANGULAR HULL 1, a DUF640 gene, is associated with grain shape, size and weight in rice," Science China. Life Sciences, vol. 56, no. 3, pp. 275283, 2013.

[24] D. Ren, Y. Rao, L. Wu et al., "The pleiotropic ABNORMAL FLOWER AND DWARF1 affects plant height, floral development and grain yield in rice," Journal of Integrative Plant Biology, vol. 58, no. 6, pp. 529-539, 2016.

[25] P. Peng, L. Liu, J. Fang, J. Zhao, S. Yuan, and X. Li, “The rice TRIANGULAR HULL1 protein acts as a transcriptional repressor in regulating lateral development of spikelet," Scientific Reports, vol. 7, no. 1, article 13712, 2017.

[26] L. Zhao, M. Nakazawa, T. Takase et al., "Overexpression of LSH1, a member of an uncharacterised gene family, causes enhanced light regulation of seedling development," Plant Journal, vol. 37, no. 5, pp. 694-706, 2004.

[27] L. M. Iyer and L. Aravind, "ALOG domains: provenance of plant homeotic and developmental regulators from the DNA-binding domain of a novel class of DIRS1-type retroposons," Biology Direct, vol. 7, no. 1, p. 39, 2012.

[28] A. Yoshida, M. Sasao, N. Yasuno et al., "TAWAWA1, a regulator of rice inflorescence architecture, functions through the suppression of meristem phase transition," Proceedings of the National Academy of Sciences of the United States of America, vol. 110, no. 2, pp. 767-772, 2013.
[29] T. H. Lee, H. Tang, X. Wang, and A. H. Paterson, "PGDD: a database of gene and genome duplication in plants," Nucleic Acids Research, vol. 41, pp. D1152-D1158, 2013.

[30] B. J. Haas, A. L. Delcher, J. R. Wortman, and S. L. Salzberg, "DAGchainer: a tool for mining segmental genome duplications and synteny," Bioinformatics, vol. 20, no. 18, pp. 36433646, 2004.

[31] B. S. Gaut, B. R. Morton, B. C. McCaig, and M. T. Clegg, "Substitution rate comparisons between grasses and palms: synonymous rate differences at the nuclear gene Adh parallel rate differences at the plastid gene rbcL," Proceedings of the National Academy of Sciences of the United States of America, vol. 93, no. 19, pp. 10274-10279, 1996.

[32] J. Castresana, "Selection of conserved blocks from multiple alignments for their use in phylogenetic analysis," Molecular Biology and Evolution, vol. 17, no. 4, pp. 540-552, 2000.

[33] R. C. Edgar, "MUSCLE: multiple sequence alignment with high accuracy and high throughput," Nucleic Acids Research, vol. 32, no. 5, pp. 1792-1797, 2004.

[34] S. Guindon, J. F. Dufayard, V. Lefort, M. Anisimova, W. Hordijk, and O. Gascuel, "New algorithms and methods to estimate maximum-likelihood phylogenies: assessing the performance of PhyML 3.0," Systematic Biology, vol. 59, no. 3, pp. 307-321, 2010.

[35] F. Chevenet, C. Brun, A. L. Banuls, B. Jacq, and R. Christen, "TreeDyn: towards dynamic graphics and annotations for analyses of trees," BMC Bioinformatics, vol. 7, no. 1, p. 439, 2006.

[36] B. Hu, J. Jin, A. Y. Guo, H. Zhang, J. Luo, and G. Gao, "GSDS 2.0: an upgraded gene feature visualization server," Bioinformatics, vol. 31, no. 8, pp. 1296-1297, 2015.

[37] T. L. Bailey, M. Boden, F. A. Buske et al., "MEME SUITE: tools for motif discovery and searching," Nucleic Acids Research, vol. 37, pp. W202-W208, 2009.

[38] L. Wang, W. Xie, Y. Chen et al., "A dynamic gene expression atlas covering the entire life cycle of rice," Plant Journal, vol. 61, no. 5, pp. 752-766, 2010.

[39] S. Dash, J. Van Hemert, L. Hong, R. P. Wise, and J. A. Dickerson, "PLEXdb: gene expression resources for plants and plant pathogens," Nucleic Acids Research, vol. 40, no. D1, pp. D1194-D1201, 2011.

[40] S. Brenner, M. Johnson, J. Bridgham et al., "Gene expression analysis by massively parallel signature sequencing (MPSS) on microbead arrays," Nature Biotechnology, vol. 18, no. 6, pp. 630-634, 2000.

[41] M. Schena, D. Shalon, R. W. Davis, and P. O. Brown, "Quantitative monitoring of gene expression patterns with a complementary DNA microarray," Science, vol. 270, no. 5235, pp. 467-470, 1995.

[42] J. Yu, J. Wang, W. Lin et al., "The genomes of Oryza sativa: a history of duplications," PLoS Biology, vol. 3, no. 2, article e38, 2005.

[43] K. H. Wolfe, M. Gouy, Y. W. Yang, P. M. Sharp, and W. H. Li, "Date of the monocot-dicot divergence estimated from chloroplast DNA sequence data," Proceedings of the National Academy of Sciences of the United States of America, vol. 86, no. 16, pp. 6201-6205, 1989.

[44] D. S. Sato, Y. Ohmori, H. Nagashima, T. Toriba, and H. Y. Hirano, "A role for TRIANGULAR HULL1 in fine-tuning spikelet morphogenesis in rice," Genes \& Genetic Systems, vol. 89, no. 2, pp. 61-69, 2014. 
[45] I. Khanday, S. R. Yadav, and U. Vijayraghavan, "Rice LHS1/OsMADS1 controls floret meristem specification by coordinated regulation of transcription factors and hormone signaling pathways," Plant Physiology, vol. 161, no. 4, pp. 1970-1983, 2013.

[46] Y. Hu, W. Liang, C. Yin et al., "Interactions of OsMADS1 with floral homeotic genes in rice flower development," Molecular Plant, vol. 8, no. 9, pp. 1366-1384, 2015.

[47] S. Lee, S. C. Choi, and G. An, "Rice SVP-group MADS-box proteins, OsMADS22 and OsMADS55, are negative regulators of brassinosteroid responses," Plant Journal, vol. 54, no. 1, pp. 93-105, 2008.

[48] S. Lee, J. Kim, J. J. Han, M. J. Han, and G. An, "Functional analyses of the flowering time gene OsMADS50, the putative SUPPRESSOR OF OVEREXPRESSION OF CO 1/AGAMOUS-LIKE 20 (SOC1/AGL20) ortholog in rice," Plant Journal, vol. 38, no. 5, pp. 754-764, 2004.

[49] E. Cho and P. C. Zambryski, "Organ boundaryl defines a gene expressed at the junction between the shoot apical meristem and lateral organs," Proceedings of the National Academy of Sciences of the United States of America, vol. 108, no. 5, pp. 2154-2159, 2011.

[50] S. Takeda, K. Hanano, A. Kariya et al., "CUP-SHAPED COTYLEDON1 transcription factor activates the expression of LSH4 and LSH3, two members of the ALOG gene family, in shoot organ boundary cells," Plant Journal, vol. 66, no. 6, pp. 1066-1077, 2011.

[51] M. O. Press and C. Queitsch, "Variability in a short tandem repeat mediates complex epistatic interactions in Arabidopsis thaliana," Genetics, vol. 205, no. 1, pp. 455-464, 2017.

[52] C. A. MacAlister, S. J. Park, K. Jiang et al., "Synchronization of the flowering transition by the tomato TERMINATING FLOWER gene," Nature Genetics, vol. 44, no. 12, pp. 13931398, 2012.

[53] S. W. Roy and D. Penny, "Patterns of intron loss and gain in plants: intron loss-dominated evolution and genome-wide comparison of O. sativa and A. thaliana," Molecular Biology and Evolution, vol. 24, no. 1, pp. 171-181, 2007.

[54] M. Y. Ma, T. Zhu, X. N. Li et al., "Imprecise intron losses are less frequent than precise intron losses but are not rare in plants," Biology Direct, vol. 10, no. 1, p. 24, 2015.

[55] R. Arora, P. Agarwal, S. Ray et al., "MADS-box gene family in rice: genome-wide identification, organization and expression profiling during reproductive development and stress," $B M C$ Genomics, vol. 8, no. 1, p. 242, 2007.

[56] G. N. Khong, P. K. Pati, F. Richaud et al., "OsMADS26 negatively regulates resistance to pathogens and drought tolerance in rice," Plant Physiology, vol. 169, no. 4, pp. 2935-2949, 2015.

[57] C. Yu, Y. Liu, A. Zhang et al., "MADS-box transcription factor OsMADS25 regulates root development through affection of nitrate accumulation in rice," PLoS One, vol. 10, no. 8, article e0135196, 2015.

[58] J. Tao, W. Liang, G. An, and D. Zhang, "OsMADS6 controls flower development by activating rice FACTOR OF DNA METHYLATION LIKE1," Plant Physiology, vol. 177, no. 2, pp. 713-727, 2018.

[59] K. Prasad, S. Parameswaran, and U. Vijayraghavan, "OsMADS1, a rice MADS-box factor, controls differentiation of specific cell types in the lemma and palea and is an early-acting regulator of inner floral organs," Plant Journal, vol. 43, no. 6, pp. 915-928, 2005.
[60] Z. Dai, J. Wang, M. Zhu, X. Miao, and Z. Shi, “OsMADS1 represses microRNA172 in elongation of palea/lemma development in rice," Frontiers in Plant Science, vol. 7, p. 1891, 2016.

[61] K. Wang, D. Tang, L. Hong et al., "DEP and $A F O$ regulate reproductive habit in rice," PLoS Genetics, vol. 6, no. 1, article e1000818, 2010.

[62] X. Gao, W. Liang, C. Yin et al., "The SEPALLATA-like gene OsMADS34 is required for rice inflorescence and spikelet development," Plant Physiology, vol. 153, no. 2, pp. 728 $740,2010$.

[63] X. Lin, F. Wu, X. du et al., "The pleiotropic SEPALLATA-like gene OsMADS34 reveals that the 'empty glumes' of rice (Oryza sativa) spikelets are in fact rudimentary lemmas," New Phytologist, vol. 202, no. 2, pp. 689-702, 2014.

[64] Y. Shinozaki, P. Nicolas, N. Fernandez-Pozo et al., "Highresolution spatiotemporal transcriptome mapping of tomato fruit development and ripening," Nature Communications, vol. 9, no. 1, p. 364, 2018.

[65] C. Liu, X. Qu, Y. Zhou et al., "OsPRR37 confers an expanded regulation of the diurnal rhythms of the transcriptome and photoperiodic flowering pathways in rice," Plant, Cell \& Environment, vol. 41, no. 3, pp. 630-645, 2018. 


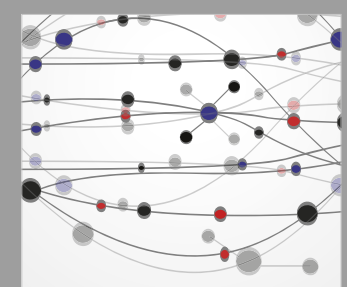

The Scientific World Journal
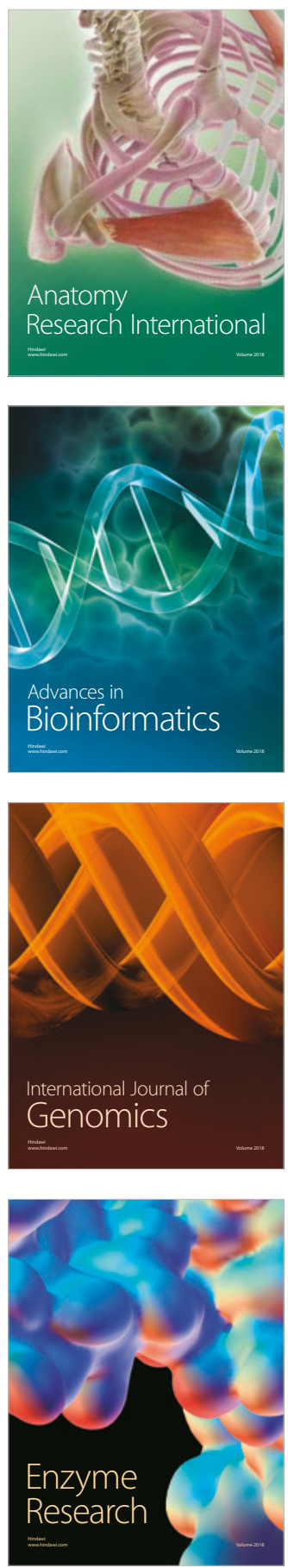
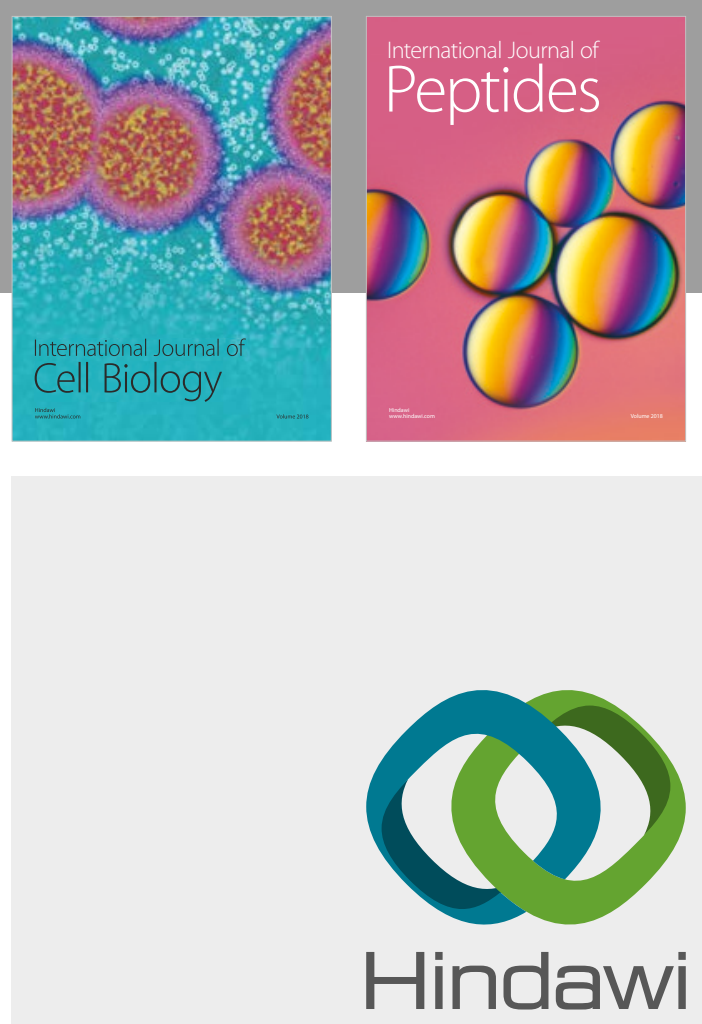

Submit your manuscripts at

www.hindawi.com
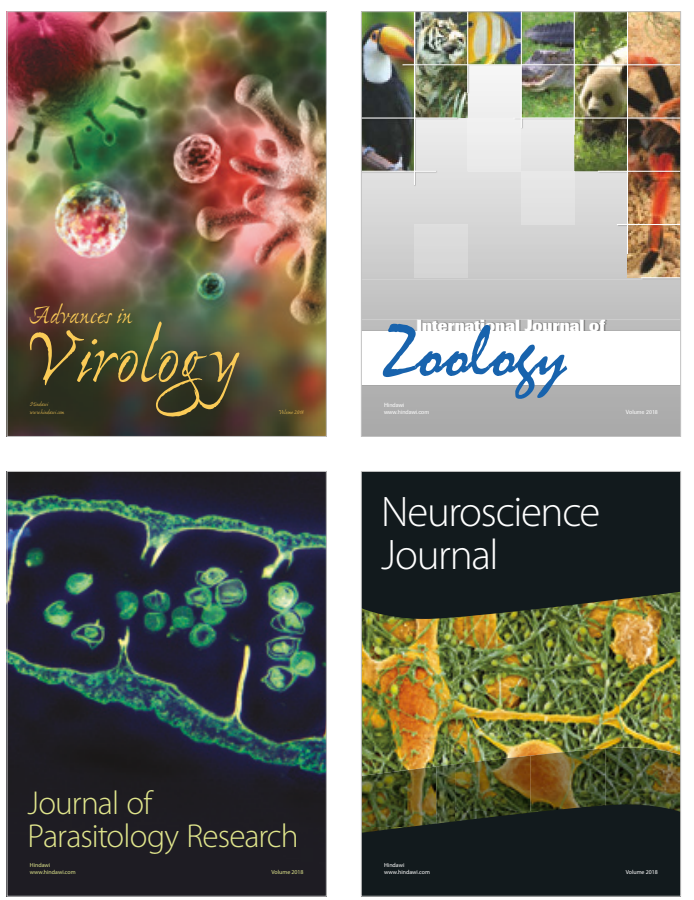
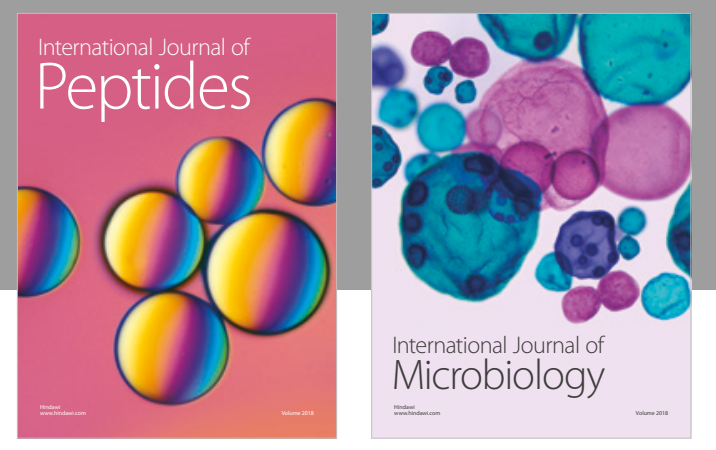

nternational Journal of Microbiology
Journal of
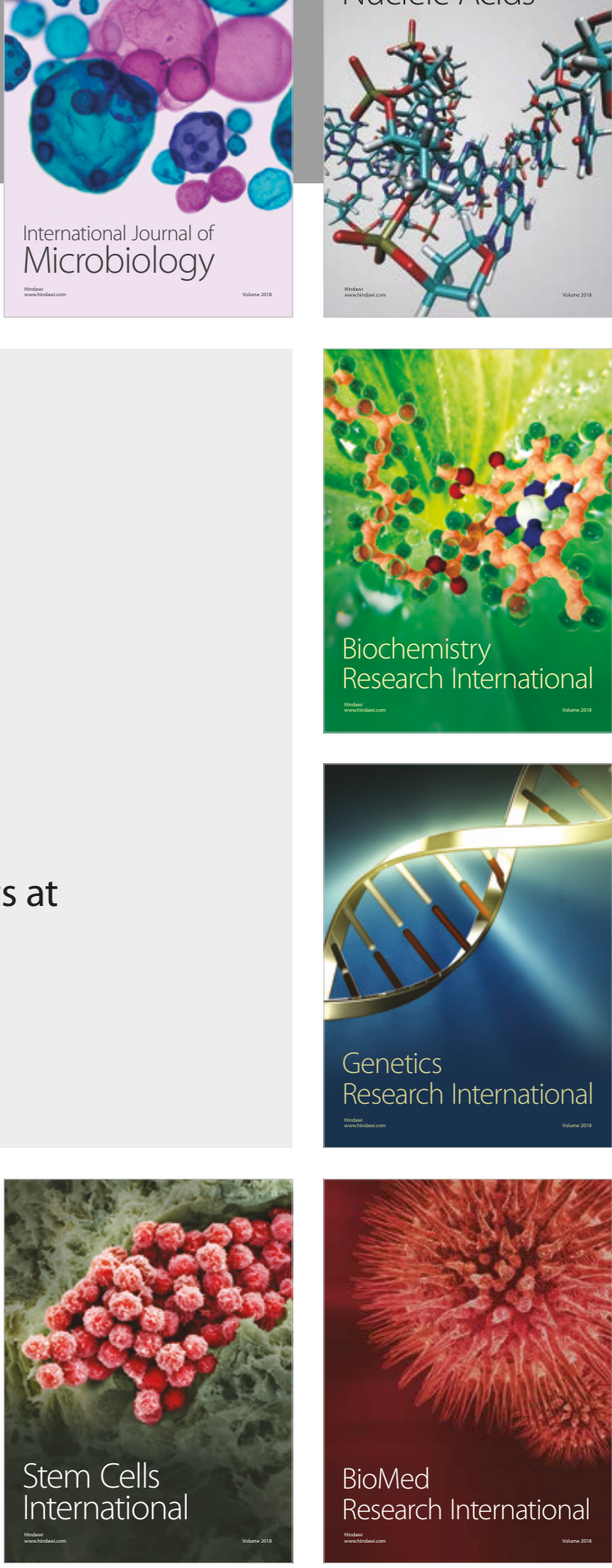
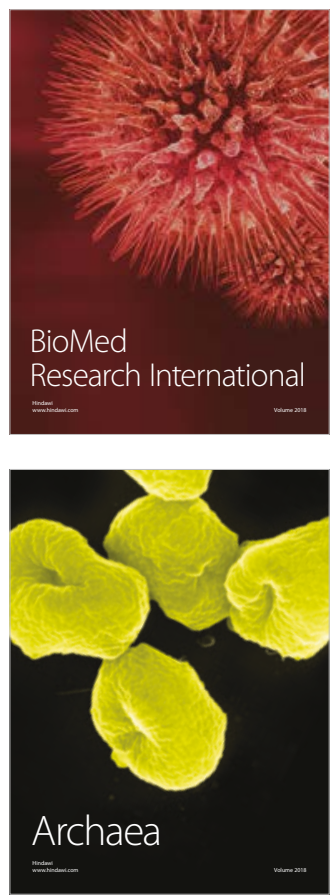